\title{
Clinical Assessment of Dialysis Recovery Time and Symptom Burden: Impact of Switching Hemodialysis Therapy Mode
}

\author{
Stephanie Bolton' \\ Rachel Gair ${ }^{2}$ \\ Lars-Göran Nilsson (iD ${ }^{3}$ \\ Michael Matthews' \\ Louanne Stewart' \\ Natasha McCullagh' \\ 'Renal Unit, Antrim Area Hospital, \\ Northern Health and Social Care Trust, \\ Northern Ireland, UK; ${ }^{2}$ UK Renal \\ Registry, Bristol, UK; ${ }^{3}$ Baxter \\ International Inc., Lund, Sweden
}

Introduction: Most people on hemodialysis (HD) report a high symptom burden. Fatigue and lack of energy are prominent, interfering with daily life and associated with poor outcome. Prolonged recovery time after each of the thrice weekly dialysis treatments is common. The impact of HD therapies, like expanded hemodialysis (HDx), on patient reported recovery time and symptom burden is unclear.

Methods: A dialysis unit decided to perform regular assessments of patient-reported symptom burden, using the POS-S Renal Symptom questionnaire and the "Recovery time from last dialysis session" question as part of routine patient focused care. At a similar time, a clinical evidence-based decision was taken to switch the in-center dialysis cohort from regular high-flux dialysis membrane to medium cut-off (MCO) membrane, introducing HDx therapy.

Results: Quarterly assessment of patient-reported symptom burden was well accepted. A sustained clinically relevant reduction in post-dialysis recovery time was observed following the therapy switch. In patients providing data up to 12 months $(\mathrm{N}=58)$, median recovery time decreased from $210 \mathrm{~min}(\mathrm{IQR} 7.5-600)$ to $60 \mathrm{~min}(0-210 ; \mathrm{p}=0.002)$ and $105 \mathrm{~min}(0$ $180 ; \mathrm{p}=0.001)$ at 6 and 12 months, respectively. Thirty-six percent of individuals reported a recovery time longer than 360 minutes at the initial assessment, which decreased to $9 \%$ at 12 months. The POS-S Renal total symptom score showed a decrease at 6 months but no difference from baseline at 12 months. The "fatigue/lack of energy" symptom showed a sustained improvement; the percentage of participants scoring its impact as "severe" or "overwhelming" decreased from $28 \%$ at baseline to $16 \%$ at 12 months. Changes in other symptoms were more variable.

Conclusion: Regular assessment of patient reported symptoms is feasible in routine dialysis practice and can help in evaluating the impact of clinical interventions. Observations suggest that HDx therapy may reduce post-dialysis recovery time and improve perceived fatigue level.

Keywords: post-dialysis recovery time, symptom burden, fatigue, HDx therapy

\section{Introduction}

People with end-stage renal disease on hemodialysis (HD) show a high symptom burden affecting their quality of life. ${ }^{1,2}$ Fatigue and lack of energy are common, causing distress, interfering with daily life, and are also associated with mortality. ${ }^{3}$ The Standardized Outcomes in Nephrology for HD (SONG-HD) workshop identified fatigue as a core outcome to assess when evaluating changes in dialysis care. ${ }^{4}$ Post-dialysis fatigue is a special issue that may substantially impair quality of life
Correspondence: Stephanie Bolton Renal Unit, Antrim Area Hospital, Northern Health and Social Care Trust. Northern Ireland, UK

Email Stephanie.Bolton@northerntrust. hscni.net 
for many HD individuals. ${ }^{5}$ Individuals on conventional HD typically report a median post-dialysis recovery time (DRT) in the range of 2-4 hours with approximately $25 \%$ reporting a recovery time exceeding 6 hours ${ }^{6,7}$ while patients on daily or nocturnal HD report a substantially shorter recovery time. ${ }^{8}$ A good understanding of the HD patient's perceived level of fatigue and overall symptom burden is essential when developing a more personcentered approach to dialysis care, and several patientreported outcome measures (PROMs) are available and may be used in regular clinical practice. ${ }^{9}$ Furthermore, examining PROMs gives additional important evidence when instigating technical changes in dialysis therapy delivery.

The last decades have seen conversion to high-flux membranes for the delivery of HD treatments and increasing use of on-line hemodiafiltration (HDF), resulting in improved clearance of middle molecule uremic toxins (MMs). However, a change from HD to HDF did not result in a sustained reduction in intra-dialytic symptoms. ${ }^{10}$ The CONTRAST study, comparing HDF to low-flux HD, found similar decline in health-related quality of life over time with both modalities; ${ }^{11}$ post-dialysis fatigue was not improved by HDF in comparison to high-flux HD in another randomized study. ${ }^{12}$

Large MMs $(>15 \mathrm{kDa})$ are generally not effectively removed by high-flux membranes. Interleukin-6 (IL-6) and other cytokines, free immunoglobulin light chains, and several other proteins belong to this group of uremic solutes that in chronic kidney disease appear to play an important role in cardiovascular disease, immunodeficiency, and malnutrition. ${ }^{13,14}$ Less is known about their role in patientreported outcome measures. It has been proposed that the retention of large MMs in people with chronic kidney disease could influence the symptom burden, for example by driving disease processes that contribute to the symptom burden and by directly causing symptoms; for example enhanced removal of a1-microglobulin is associated with improvement in restless leg syndrome. ${ }^{15,16}$ A medium cut-off (MCO) membrane was recently introduced, allowing for expanded hemodialysis (HDx) to achieve more effective clearance of large MMs, even when compared to HDF. ${ }^{17,18}$ Safe application of the HDx therapy was confirmed in controlled studies, ${ }^{19-22}$ while long-term outcome data for HDx in comparison to HD or HDF are still scarce. A large one-year prospective observational study indicated that peoples' quality of life was improved with HDx therapy, ${ }^{23}$ but further studies are needed to assess the impact of enhanced dialytic removal of large MMs on the dialysis-related symptom burden.
The multi-professional team at the Antrim Renal Unit took a decision in 2018 to implement a PROM program to capture HD patients' symptom burden as part of routine clinical care. In parallel the team made an evidence-based decision to implement HDx, using the MCO membrane, as the preferred in-center hemodialysis therapy to achieve effective MM clearance. Before, HDF had been considered the preferred hemodialysis therapy for most of the dialysis cohort but achieving a high enough convective volume in HDF was found difficult in many cases as the blood flow rate was limited by a poorly functioning arterio-venous fistula (AVF) and a necessity in many patients to use a catheter for blood access. By the beginning of 2018 only $28 \%$ of the prevalent individuals at the Antrim in-center unit were considered suitable for HDF. This paper reports on the initial 12month experience from applying HDx therapy, focusing on the patient-reported symptom burden during this period.

\section{Materials and Methods PROM Data Collections}

PROM data collection started in the period from March 8 to April 18, 2018 and were thereafter performed at quarterly intervals. PROM assessments were typically administered to people while they were on dialysis, mostly at a mid-week session. Individuals were asked about their post-dialysis recovery time using the question "How long does it take to get back to normal, after dialysis?". 8 Data on symptom prevalence and severity were collected using the 17-item version of the Palliative Care Outcome Scale-Symptom module for renal patients (POS-S Renal), ${ }^{24,25}$ which asks how 17 predefined symptoms had affected patients in the past week using a 5-point scale from "not at all" to "overwhelmingly".

\section{Dialysis Treatments}

At the start of the PROM data collection individuals were on conventional thrice weekly hemodialysis at the Antrim incenter dialysis unit and treated with regular high-flux membranes (Revaclear dialyzer in HD and Polyflux H dialyzer in HDF; Baxter Healthcare Ltd). Following the implementation of PROM assessments, the Antrim renal team made an evidence-based decision to implement HDx using the MCO membrane (Theranova dialyzer; Baxter Healthcare Ltd). Treatment prescription factors such as frequency, blood flow rate (median $300 \mathrm{~mL} / \mathrm{min}$ ), dialysate flow $(500 \mathrm{~mL} /$ min), and treatment time (median 4 hours) were not affected by the therapy change. Actual times for PROM data 
collections were $95 \pm 10,204 \pm 10,300 \pm 14$, and $362 \pm 9$ days following the switch to the MCO membrane.

\section{Statistical Analysis}

Post-dialysis recovery time in minutes was for each time point summarized as median and interquartile range (IQR) with differences between baseline and later stages analyzed by the Wilcoxon signed-rank test. The POS-S Renal survey results are reported for individual symptoms as well as through a total score derived by summing up for each patient the individual scores ("not at all" $=0$, "slightly" $=1$, "moderately" $=2$, "severely" $=3$, "overwhelmingly" $=4$ ), giving a final score between 0 and 68. Results were summarized as median and IQR with differences between baseline and later stages analyzed by the Wilcoxon signed-rank test.

\section{Results}

Initially, 90 people agreed to provide PROMs data; the baseline characteristics of these are given in Table 1 . An additional 20 individuals were either unable to provide PROMs data or did not consent to do it. Participant numbers providing data at 3, 6, 9, and 12 months were 80, 72, 68, and 59 respectively. Fourteen individuals died during the 12-month survey period. Other reasons for dropout were transplantation $(\mathrm{N}=3)$, decline to participate $(\mathrm{N}=8)$, development of confusion making them unable to respond $(\mathrm{N}=3)$, transfer to home $\mathrm{HD}(\mathrm{N}=1)$ or to another unit $(\mathrm{N}=1)$, and becoming dialysis independent $(\mathrm{N}=1)$. The $\mathrm{MCO}$ membrane was introduced without incident over a 2-week period to all individuals on $\mathrm{HD}$ at the Antrim in-center dialysis unit $(\mathrm{N}=110)$ and has remained standard of care. All individuals tolerated the new membrane well. No clinically significant changes in albumin, C-reactive protein, and hemoglobin levels became apparent. No signs of increased infection rate were observed.

\section{Post-Dialysis Recovery Time}

The median self-reported recovery time at baseline was 240 min (IQR: $60-720 ; \mathrm{N}=89$ ). At follow-up, the recovery time was shorter: $120 \mathrm{~min}$ (22-435), $60 \mathrm{~min}(0-240), 60 \mathrm{~min}(0$ 240), and $105 \min (0-180)$ at 3, 6, 9, and 12 months,
Table I Baseline Demographics for Individuals Contributing with Patient-Reported Outcome Data $(\mathrm{N}=90)$

\begin{tabular}{|l|l|}
\hline Variable & Distribution \\
\hline Age, mean (SD) & $73(12)$ years \\
Gender & $61 \%$ male, 39\% female \\
\hline Primary renal disease & $43 \%$ diabetic nephropathy \\
& $10 \%$ cardiovascular disease \\
& $11 \%$ glomerulonephritis \\
& $36 \%$ others \\
\hline HD vintage & $9 \%<1$ year \\
& $53 \%$ I-3 years \\
& $11 \% 3-5$ years \\
& $18 \% 5-10$ years \\
& $6 \% 10-15$ years \\
& $3 \%>15$ years \\
\hline Vascular access & $48 \%$ arterio-venous fistula \\
& $1 \%$ arterio-venous graft \\
& $51 \%$ catheter \\
\hline HDF, N (\%) & 25 (28\%) \\
\hline
\end{tabular}

respectively, with differences between baseline and the 6-, 9-, and 12-month ratings being statistically significant at $\mathrm{p}<$ 0.01 . The subgroup of participants who provided recovery time data throughout the 12-month period $(\mathrm{N}=58)$ reported a similarly decreased recovery time (Table 2 and Figure 1). In

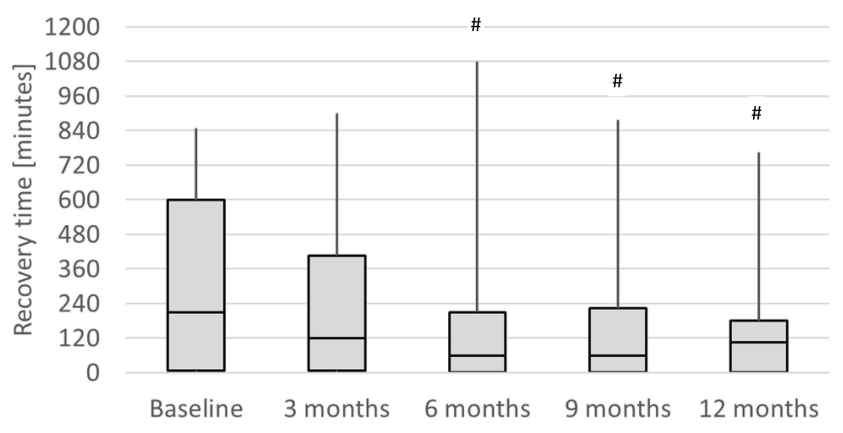

Figure I Reported post-dialysis recovery times for individuals who completed the I2-months observation period $(\mathrm{N}=58)$.

Notes: Boxes show medians and 25th/75th percentiles and whiskers show 95th percentiles. "Denotes $p<0.01$ vs baseline.

Table 2 Self-Reported Post-Dialysis Recovery Times in Minutes (Median, IQR) for Participants Who Provided Ratings UP to I2 Months $(\mathrm{N}=58)$

\begin{tabular}{|l|c|c|c|c|}
\hline Baseline & 3 Months & 6 Months & 9 Months & 12 Months \\
\hline $210(7.5-600)$ & $120(7.5-405)$ & $60(0-210)$ & $60(0-225)$ & $105(0-180)$ \\
& $P=0.2$ & $P=0.002$ & $P<0.001$ & $P=0.001$ \\
\hline
\end{tabular}

Note: P-values are in comparison to baseline. 
Table 3 POS-S Renal Total Symptom Scores (Median, IQR) for Participants Who Provided Ratings Up to I2 Months (N = 56)

\begin{tabular}{|l|c|c|c|c|}
\hline Baseline & 3 Months & 6 Months & 9 Months & 12 Months \\
\hline $12(7-17.3)$ & $10(4.5-16)$ & $8(5-19)$ & $11(5.8-20.3)$ & $12(5-22)$ \\
& $P=0.06$ & $P=0.003$ & $P=0.8$ & $P=0.8$ \\
\hline
\end{tabular}

Note: P-values are in comparison to baseline.

this subgroup, the percentage of people reporting a recovery time greater than 360 minutes decreased from $36 \%$ at baseline to $26 \%, 14 \%, 14 \%$, and $9 \%$ at $3,6,9$, and 12 months, respectively. Individuals with vascular access through a catheter generally reported longer recovery times than those with an AVF access but improvements in recovery time during the observation period were seen regardless of access type.

\section{Symptom Burden}

At baseline, the median number of symptoms per participant was $7(I Q R 4-10 ; N=90)$ and the total symptom score varied between individuals from 1 to 42, with a median value of 13 (IQR 7-18.8). At the 3- and 6-month follow-ups, the total symptom score showed a decrease from baseline, to 11 (516) at 3 months $(\mathrm{p}=0.03)$ and $10.5(5-19)$ at 6 months $(\mathrm{p}=$ $0.005)$, while subsequent follow-ups were not different from baseline. The subgroup of participants who completed the 12-month observation period showed a baseline total score of $12(7-17.3 ; \mathrm{N}=56)$, with a significant decrease at 6 months (Table 3 ). In this subgroup, $66 \%$ of patients reported at least one symptom as severe or overwhelming at baseline, which later decreased to $35 \%, 43 \%, 48 \%$ and $54 \%$ at $3,6,9$, and 12 months, respectively. Individuals with a catheter access reported a higher total symptom score at baseline than those with AVF access while the improvements at 3 and 6 months appeared unrelated to the type of access.

"Weakness or lack of energy" and "Poor mobility" were reported as the most bothersome symptoms followed by "Sore or dry mouth", "Difficulty sleeping", and "Itching". For "Weakness or lack of energy" the percentage of patients reporting that it affected them "severely" or "overwhelmingly" in the past week decreased from $28 \%$ at baseline to $16 \%, 15 \%, 20 \%$, and $16 \%$ at $3,6,9$, and 12 months, respectively (Table 4). Other symptoms showed only minor changes during the observation period.

Table 4 POS-S Renal Symptoms and Fraction of Participants Reporting the Individual Symptom to Affect Them Severely in the Past Week ( $N=56$ Patients Who Completed the I2-Months PROM Assessment)

\begin{tabular}{|c|c|c|c|c|c|}
\hline \multirow[t]{2}{*}{ Symptom } & \multicolumn{5}{|c|}{ Fraction of Patients Scoring Symptom Impact as "Severe" or "Overwhelming" } \\
\hline & Baseline & 3 Months & 6 Months & 9 Months & 12 Months \\
\hline Poor mobility & $30 \%$ & $25 \%$ & $21 \%$ & $25 \%$ & $29 \%$ \\
\hline Weakness or lack of energy & $28 \%$ & $16 \%$ & $15 \%$ & $20 \%$ & $16 \%$ \\
\hline Sore or dry mouth & $20 \%$ & $18 \%$ & $11 \%$ & $9 \%$ & $13 \%$ \\
\hline Difficulty sleeping & $16 \%$ & $4 \%$ & $13 \%$ & $13 \%$ & $18 \%$ \\
\hline Itching & $15 \%$ & $4 \%$ & $11 \%$ & $9 \%$ & $7 \%$ \\
\hline Pain & $14 \%$ & $4 \%$ & $4 \%$ & $14 \%$ & $13 \%$ \\
\hline Shortness of breath & $13 \%$ & $6 \%$ & $8 \%$ & $5 \%$ & $13 \%$ \\
\hline Drowsiness & $9 \%$ & $8 \%$ & $8 \%$ & $13 \%$ & $9 \%$ \\
\hline Feeling depressed & $9 \%$ & $4 \%$ & $8 \%$ & $7 \%$ & $7 \%$ \\
\hline Restless legs or difficulty keeping legs still & $8 \%$ & $2 \%$ & $9 \%$ & $4 \%$ & $11 \%$ \\
\hline Feeling anxious or worried & $8 \%$ & $2 \%$ & $6 \%$ & $2 \%$ & $7 \%$ \\
\hline Changes in skin & $5 \%$ & $4 \%$ & $4 \%$ & $7 \%$ & $5 \%$ \\
\hline Constipation & $2 \%$ & $6 \%$ & $6 \%$ & $4 \%$ & $4 \%$ \\
\hline Poor appetite & $2 \%$ & $2 \%$ & $2 \%$ & $2 \%$ & $5 \%$ \\
\hline Diarrhea & $2 \%$ & $0 \%$ & $2 \%$ & $2 \%$ & $2 \%$ \\
\hline Nausea & $2 \%$ & $2 \%$ & $0 \%$ & $2 \%$ & $0 \%$ \\
\hline Vomiting & $2 \%$ & $2 \%$ & $0 \%$ & $0 \%$ & $0 \%$ \\
\hline
\end{tabular}

Note: Symptoms are ordered by prevalence at the baseline assessment. 


\section{Discussion}

While people on dialysis consider symptom relief as a top priority, there is a paucity of data on how dialysis treatment characteristics affect their symptom burden. As the Antrim HD population was switched from regular highflux membrane applied in HD/HDF to the MCO membrane applied in HDx mode, a quarterly assessment of symptom burden using standardized PROMs revealed a sustained improvement in patient-reported post-dialysis recovery time. Based on the POS-S Renal symptom questionnaire, a temporary reduction in the total symptom score was found at 3 and 6 months. Individual symptoms showed varying trends over time. However, the "weakness/lack of energy" symptom, ranking second in severity among the 17 symptom categories at baseline, showed an improvement that was sustained over 12 months.

The finding of substantial reductions in post-dialysis recovery time and fatigue appear clinically relevant as these parameters show strong associations with several quality-oflife domains. ${ }^{23}$ In a large international observational study post-dialysis recovery time was associated with rates of first hospitalization (adjusted hazard ratio [HR] per additional hour of recovery time, 1.03 ; $95 \% \mathrm{CI}, 1.02-1.04$ ) as well as with mortality (adjusted HR, 1.05; 95\% CI 1.031.07). ${ }^{6}$ The recovery question, though slightly modified, was recently proposed as a key part of the Symptom Monitoring on Renal Replacement Therapy-Hemodialysis (SMaRRT$\mathrm{HD}^{\mathrm{TM}}$ ) PROM tool to assess patient-reported outcomes. ${ }^{26}$

The high symptom burden in this HD population is like that reported in other studies using the same survey. ${ }^{27,28}$ We found for many symptoms a considerable variation in symptom severity over time with a reduced total symptom score at 3 and 6 months. While this may indicate an effect of changing to the HDx therapy, a similar reduction in symptom burden at 3 months after the initial assessment has been reported also without a change in dialysis prescription. ${ }^{28}$ The 9- and 12-month total symptom scores appeared not different from baseline scores, indicating a u-curved change in individuals' rating of their symptom burden after commencing the POS-S Renal questionnaire assessment and/or implementing a change in their HD therapy. This is an interesting observation that will need further studies to elucidate. However, we note with interest that the improvement in recovery time after the therapy change was maintained throughout the 12-month observation period.
Fatigue/lack of energy, a prominent symptom in our population, showed a consistent improvement over our 12 months' follow-up. This appears as consistent with a recent randomized clinical trial showing that the KDQOL-36 domains of physical functioning and physical role improved at 3 months with the MCO membrane compared to a high-flux membrane. ${ }^{29}$

Few data exist on how patient-reported fatigue changes over time in people on HD. Bossola et al found the vitality subscale of the SF-36 questionnaire to be stable during a 1-year course although with considerable individual variations. ${ }^{30}$ Picariello et al, studying an HD cohort in the UK annually over 3 years, found the perceived fatigue severity to be stable over time while fatigue-related functional impairment increased significantly. ${ }^{31}$

The pathophysiology behind fatigue in dialysis remains unclear. Interestingly, in other disease states emerging evidence indicate a causal link between inflammation and fatigue. ${ }^{32}$ In people on chronic HD, fatigue appears associated with the serum level of interleukin 6, supporting that inflammation plays a role. $^{33}$ Increased oxidative stress, which is prominent in $\mathrm{HD},{ }^{34}$ has also been proposed as a significant link to uremic myopathy and fatigue in renal failure. ${ }^{35}$ Uremic toxins implicated in uremia-related inflammation typically fall into the category of large middle molecules and may therefore be better cleared by the MCO membrane, ${ }^{36}$ potentially resulting in improved inflammatory and oxidative stress status. In support of such a hypothesis, a recent 3-months cross-over study found reduced levels of oxidative stress markers with HDx compared to high-flux HD. ${ }^{19}$

The importance of administering PROM surveys repeatedly over time to capture symptom fluctuations has been stressed. ${ }^{26}$ However, to our knowledge there is no consensus on the best frequency to administer different PROMs. We found a quarterly administration of the recovery time question and the POS-S Renal symptom survey to be easy and well accepted by most patients. With simpler tools such as a patient mobile application, even a daily or weekly administration can be achievable and may be preferred. ${ }^{37}$

We recognize that our findings from this pilot retrospective analysis need to be interpreted with caution. We cannot exclude that the improvements we saw in recovery time and fatigue were unrelated to the membrane and therapy change, especially as this was not a formal study with a control group. We find it unlikely, though, that post-dialysis recovery time and fatigue scoring would have decreased significantly over 
time if these patients had stayed on their regular therapy prescription. If any change at all, we would expect it to be a worsening of symptom burden with increasing HD vintage. However, it should be considered that PROM data were not returned anonymously so the identification of specific symptoms at the initial assessment could have led to improved symptom management resulting in reduced symptom severity at later stages. A strength of these findings is that they reflect a real-life assessment of PROMs in about $80 \%$ of the in-center HD population. However, being a single-center experience, these results may not necessarily be applicable to dialysis populations in other dialysis centers with other dialysis practices.

\section{Conclusion}

We found a quarterly application of PROM tools to an incenter HD population to be feasible and well accepted by patients. Sustained improvements in patient-reported postdialysis recovery time and POS-S Renal fatigue score were observed over a 12-month period, coinciding with a switch from regular HD/HDF using high-flux membranes to HDx using the MCO membrane. These observations provide indications that enhanced clearance of large middle molecules, as achieved by expanded hemodialysis, may affect HD individuals' symptom burden in a positive way. However, properly controlled studies are needed to confirm such an effect. Such studies should preferably avoid a too narrow patient selection to mirror real-life clinical practice.

\section{Ethics Statement}

This retrospective cohort analysis utilized data gathered through the Antrim Renal Unit's introduction of standard routine clinical practice of assessing HD patients' view of their dialysis-related symptom burden and a Renal Unit decision to change dialysis membrane used routinely in the clinic. Therefore, the data were gathered under UK NHS clinical audit and quality improvement governance and informed consent arrangements, so no ethical approval was required. Participation was voluntary for providing routinely collected symptom burden assessments.

\section{Acknowledgments}

We would like to express our gratitude to all individuals at the Antrim dialysis unit who responded to the applied questionnaires on their symptom burden.

\section{Disclosure}

L.G.N. is an employee of Gambro Lundia AB, a wholly owned subsidiary of Baxter International Inc. SB reports non-financial support from Baxter Healthcare for Statistical analysis and manuscript preparation and speaker fees from Baxter Europe for presenting her own work. The authors report no other conflicts of interest in this work.

\section{References}

1. Lowney AC, Myles HT, Bristowe K, et al. Understanding what influences the health-related quality of life of hemodialysis patients: a collaborative study in England and Ireland. J Pain Symptom Manag. 2015;50:778-785.

2. Flythe JE, Dorough A, Narendra JH, Forfang D, Hartwell L, AbdelRahman E. Perspectives on symptom experiences and symptom reporting among individuals on hemodialysis. Nephrol Dial Transplant. 2018;33:1842-1852.

3. Jhamb M, Argyropoulos C, Steel JL, et al. Correlates and outcomes of fatigue among incident dialysis patients. Clin J Am Soc Nephrol. 2009;4:1779-1786.

4. Evangelidis N, Tong A, Manns B, et al. Developing a set of core outcomes for trials in hemodialysis: an international Delphi survey. Am J Kidney Dis. 2017;70:464-475.

5. Bossola M, Tazza L. Postdialysis fatigue: a frequent and debilitating symptom. Semin Dial. 2016;29:222-227.

6. Rayner HC, Zepel L, Fuller DS, et al. Recovery time, quality of life, and mortality in hemodialysis patients: the Dialysis Outcomes and Practice Patterns Study (DOPPS). Am J Kidney Dis. 2014;64:86-94.

7. Alvarez L, Brown D, Hu D, Chertow GM, Vassalotti JA, Prichard S. Intradialytic symptoms and recovery time in patients on thrice-weekly in-center hemodialysis: a cross-sectional online survey. Kidney Med. 2019;2:125-130.

8. Lindsay RM, Heidenheim PA, Nesrallah G, et al. Minutes to recovery after a hemodialysis session: a simple health-related quality of life question that is reliable, valid, and sensitive to change. Clin J Am Soc Nephrol. 2006;1:952-959.

9. Aiyegbusi OL, Kyte D, Cockwell P, et al. Measurement properties of patient-reported outcome measures (PROMs) used in adult patients with chronic kidney disease: a systematic review. PLoS One. 2017;12:e0179733.

10. Caplin B, Alston H, Davenport A. Does online hemodiafiltration reduce intra-dialytic patient symptoms? Nephron Clin Pract. 2013;124:184-190.

11. Mazairac AH, de Wit GA, Grooteman MP, et al. Effect of hemodiafiltration on quality of life over time. Clin J Am Soc Nephrol. 2013;8:82-89.

12. Smith JR, Zimmer N, Bell E, Francq BG, McConnachie A, Mactier R. A randomized, single-blind, crossover trial of recovery time in high-flux hemodialysis and hemodiafiltration. Am J Kidney Dis. 2017;69:762-770.

13. Chmielewski M, Cohen G, Wiecek A, Jesús Carrero J. The peptidic middle molecules: is molecular weight doing the trick? Semin Nephrol. 2014;34:118-134.

14. Wolley M, Jardine M, Hutchison CA. Exploring the clinical relevance of providing increased removal of large middle molecules. Clin J Am Soc Nephrol. 2018;13:805-814.

15. Hutchison C, Wolley M. The rationale for expanded hemodialysis therapy (HDx). Contrib Nephrol. 2017;191:142-152.

16. Sakurai K. Biomarkers for evaluation of clinical outcomes of hemodiafiltration. Blood Purif. 2013;35(suppl 1):64-68. 
17. Ronco C. The rise of expanded hemodialysis. Blood Purif. 2017;44: I-VIII.

18. Kirsch AH, Lyko R, Nilsson LG, et al. Performance of hemodialysis with novel medium cut-off dialyzers. Nephrol Dial Transplant. 2017;32:165-172.

19. Belmouaz M, Bauwens M, Hauet T, et al. Comparison of the removal of uraemic toxins with medium cut-off and high-flux dialysers: a randomized clinical trial. Nephrol Dial Transplant. 2020;35:328-335.

20. Krishnasamy R, Hawley CM, Jardine MJ, et al. A trial evaluating mid cut-off value membrane clearance of albumin and light chains in hemodialysis patients: a safety device study. Blood Purif. 2020;49:468-478.

21. Weiner DE, Falzon L, Skoufos L, et al. Efficacy and safety of expanded hemodialysis with the Theranova 400 dialyzer: a randomized controlled trial. Clin $J$ Am Soc Nephrol. 2020;15:1310-1319.

22. Bunch A, Sanchez R, Nilsson LG, et al. Medium cut-off dialyzers in a large population of hemodialysis patients in Colombia: COREXH registry. Ther Apher Dial. 2021;25:33-43.

23. Alarcon JC, Bunch A, Ardila F, et al. Impact of medium cut-off dialyzers on patient-reported outcomes: COREXH registry. Blood Purif. 2021;50:110-118.

24. Murphy EL, Murtagh FE, Carey I, Sheerin NS. Understanding symptoms in patients with advanced chronic kidney disease managed without dialysis: use of a short patient-completed assessment tool. Nephron Clin Pract. 2009;111:c74-80.

25. Raj R, Ahuja K, Frandsen M, Murtagh FEM, Jose M. Validation of the IPOS-Renal Symptom Survey in advanced kidney disease: a cross-sectional study. J Pain Symptom Manag. 2018;56:281-287.

26. Flythe JE, Dorough A, Narendra JH, Wingard RL, Dalrymple LS, DeWalt DA. Development and content validity of a hemodialysis symptom patient-reported outcome measure. Qual Life Res. 2019;28:253-265.

27. Sexton DJ, Lowney AC, O'Seaghdha CM, et al. Do patient-reported measures of symptoms and health status predict mortality in hemodialysis? An assessment of POS-S Renal and EQ-5D. Hemodial Int. 2016;20:618-630.
28. Moskovitch JT, Mount PF, Davies MRP. Changes in symptom burden in dialysis patients assessed using a symptom-reporting questionnaire in clinic. $J$ Palliat Care. 2020;35:59-65.

29. Lim JH, Park Y, Jeong KH, et al. Randomized controlled trial of medium cut-off versus high-flux dialyzers on quality of life outcomes in maintenance hemodialysis patients. Sci Rep. 2020;10:7780.

30. Bossola M, Di Stasio E, Antocicco M, Pepe G, Marzetti E, Vulpio C. 1-year course of fatigue in patients on chronic hemodialysis. Int Urol Nephrol. 2017;49:727-734.

31. Picariello F, Norton S, Moss-Morris R, Macdougall IC, Chilcot J. A prospective study of fatigue trajectories among in-centre hemodialysis patients. Br J Health Psychol. 2020;25:61-88.

32. Dantzer R, Heijnen CJ, Kavelaars A, Laye S, Capuron L. The neuroimmune basis of fatigue. Trends Neurosci. 2014;37:39-46.

33. Bossola M, Di Stasio E, Giungi S, Rosa F, Tazza L. Fatigue is associated with serum interleukin-6 levels and symptoms of depression in patients on chronic hemodialysis. J Pain Symptom Manag. 2015;49:578-585.

34. Liakopoulos V, Roumeliotis S, Gorny X, Dounousi E, Mertens PR. Oxidative stress in hemodialysis patients: a review of the literature. Oxid Med Cell Longev. 2017;2017:3494867.

35. Kaltsatou A, Sakkas GK, Poulianiti KP, et al. Uremic myopathy: is oxidative stress implicated in muscle dysfunction in uremia? Front Physiol. 2015;6:102

36. Mitra S, Kharbanda K. Effects of expanded hemodialysis therapy on clinical outcomes. Contrib Nephrol. 2017;191:188-199.

37. Pittman ZC, John SG, McIntyre CW. Collection of daily patient reported outcomes is feasible and demonstrates differential patient experience in chronic kidney disease. Hemodial Int. 2017;21:265-267.
Patient Related Outcome Measures

\section{Publish your work in this journal}

Patient Related Outcome Measures is an international, peer-reviewed, open access journal focusing on treatment outcomes specifically relevant to patients. All aspects of patient care are addressed within the journal and practitioners from all disciplines are invited to submit their work as well as healthcare researchers and patient support groups.
The manuscript management system is completely online and includes a very quick and fair peer-review system. Visit http://www. dovepress.com/testimonials.php to read real quotes from published authors. 